\title{
The relation between the properties of the NLR in narrow-line Seyfert 1 galaxies and the accretion rate
}

\author{
Dawei $\mathrm{Xu}^{1}$ and Stefanie Komossa ${ }^{2}$ \\ ${ }^{1}$ National Astronomical Observatories, Chinese Academy of Sciences, Beijing 100012, China \\ ${ }^{2}$ Max-Planck-Institut für Extraterrestrische Physik, Postfach 1312, 85741 Garching, Germany \\ email:dwxu@bao.ac.cn, skomossa@mpe.mpg.de
}

\begin{abstract}
We present a systematic study of the properties of the narrow-line region (NLR) of narrow-line Seyfert 1 galaxies (NLS1s) using Sloan Digital Sky Survey (SDSS) spectroscopy. Various correlations between the observed parameters and physical properties of NLS1s and broad-line Seyfert 1 galaxies (BLS1s) are detected. We search for possible origins of these trends by employing correlation analyses. We further investigate the relationship between black hole mass, Eddington ratio $\left(L / L_{\mathrm{Edd}}\right)$ and physical parameters of the NLR.
\end{abstract}

\section{Introduction}

NLS1s are intriguing due to their extreme emission line and continuum properties. They lie at one extreme end of the Boroson \& Green (1992) eigenvector 1 (EV1) which governs the correlations between AGN properties. Boroson (2002) and Sulentic et al. (2003) suggested that EV1 is an indicator of $L / L_{\mathrm{Edd}}$. Density of an out-flowing wind was speculated to be among the other main drivers underlying EV1 (e.g. Lawrence et al. 1997). However, there has been little investigation on NLR density for NLS1s. We present for the first time the NLR density measurements for one of the largest homogeneously analyzed NLS1 sample to date and compare it with that of BLS1s.

\section{Sample selection}

We homogeneously analyze and compare optical emission line properties of a large number of NLS1s with BLS1s based on spectra obtained in the course of the SDSS Data Release 3 (Abazajian et al. 2005). Only objects with redshift $z$ less than 0.3 are taken into account. In order to get an accurate measurement, we require the density-sensitive ratio [S II] $\lambda \lambda 6716,6731$ to have a signal-to-noise ratio $(\mathrm{S} / \mathrm{N})$ greater than 5 . A total of 61 objects with FWHM of the broad component of $\mathrm{H} \beta$ (hereafter $\mathrm{H} \beta_{\mathrm{b}}$ ) $\leqslant 2000 \mathrm{~km} \mathrm{~s}^{-1}$ are included in the NLS1 sample, while 40 with $\operatorname{FWHM}\left(\mathrm{H} \beta_{\mathrm{b}}\right)>2000 \mathrm{~km} \mathrm{~s}^{-1}$ are included in the BLS1 sample.

\section{The density of the NLR}

One of our main goals is to examine whether or not there is a difference in electron density $n_{e}$ between NLS1s and BLS1s, in order to test different NLS1 models. We use the density diagnostic $[\mathrm{S} \mathrm{II}] \lambda 6716 / \lambda 6731$ to measure the NLR electron density. We find the sources do not homogeneously populate the $n_{e}-\operatorname{FWHM}\left(\mathrm{H} \beta_{\mathrm{b}}\right)$ diagram (Figure 1$)$. Our key finding is the detection of a 'zone of avoidance' in the $n_{e}-\mathrm{FWHM}\left(\mathrm{H} \beta_{\mathrm{b}}\right)$ diagram: BLS1s (FWHM H $\beta_{\mathrm{b}}>2000 \mathrm{~km} \mathrm{~s}^{-1}$ ) avoid low average densities, and all show $n_{e}>$ 


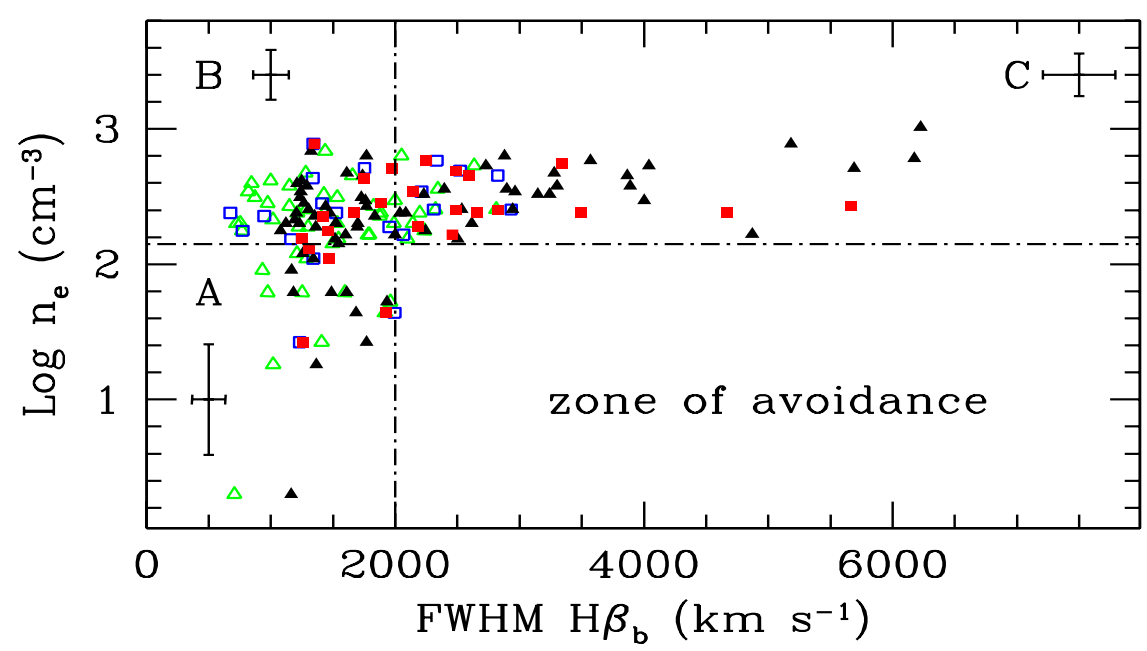

Figure 1. Electron density in dependence of FWHM of $\mathrm{H} \beta_{\mathrm{b}}$. Filled and open symbols represent $\mathrm{H} \beta_{\mathrm{b}}$ modeled by Gaussian and Lorentzian profiles, respectively. Squares are QSOs; triangles are Seyfert 1s. The dot-dashed lines distinguish areas populated by: (A) NLS1s with small $\mathrm{H} \beta_{\mathrm{b}}$ width and low density; (B) NLS1s small $\mathrm{H} \beta_{\mathrm{b}}$ width and high density; and (C) BLS1s with large $\mathrm{H} \beta_{\mathrm{b}}$ width and high density. Median error bars of each regime are given.

$140 \mathrm{~cm}^{-3}$. On the other hand, NLS1s show a larger scatter in densities in the range $n_{e}=2 \sim 770 \mathrm{~cm}^{-3}$, including a significant number of objects with low densities (see details in $\mathrm{Xu}$ et al. 2006).

In order to search for possible origins of this peculiar trend, we investigate the relationship between black hole mass, $L / L_{\mathrm{Edd}}$ and physical parameters of the NLR. The correlation analyses show that the $L / L_{\text {Edd }}$ anti-correlates with density. We investigate a number of different models for the 'zone of avoidance' in density. We tentatively favor winds/outflows and/or different host galaxy (ISM) properties.

\section{Acknowledgements}

We thank the Chinese National Science Foundation (NSF) for support under grant NSFC-10503005. DX acknowledges Max-Planck-Institut für extraterrestrische Physik and Max-Planck-Gesellschaft for financial support. This research made use of the SDSS archives and the Catalogue of Quasars and Active Nuclei.

\section{References}

Abazajian, K., Adelman-McCarthy, J. K., Agüeros, M. A. et al. 2005, AJ, 129, 1755

Boroson, T. A. \& Green, R. F. 1992, ApJS, 80, 109

Boroson, T. A. 2002, ApJ, 565, 78

Sulentic, J. W., Zamfir, S., Marziani, P., Bachev, R., Calvani, M. \& Dultzin-Hacyan, D. 2003, ApJ, 597, L17

Xu, D., Komossa, S., Zhou, H., Wang, T. \& Wei, J. 2006, ApJ, to be submitted 